\title{
Entre femmes
}

\section{La tradition de l'ichigenkin : une responsabilité de femmes}

\section{Yoshiko Okazaki}

Traducteur : Isabelle Schulte-Tenckhoff

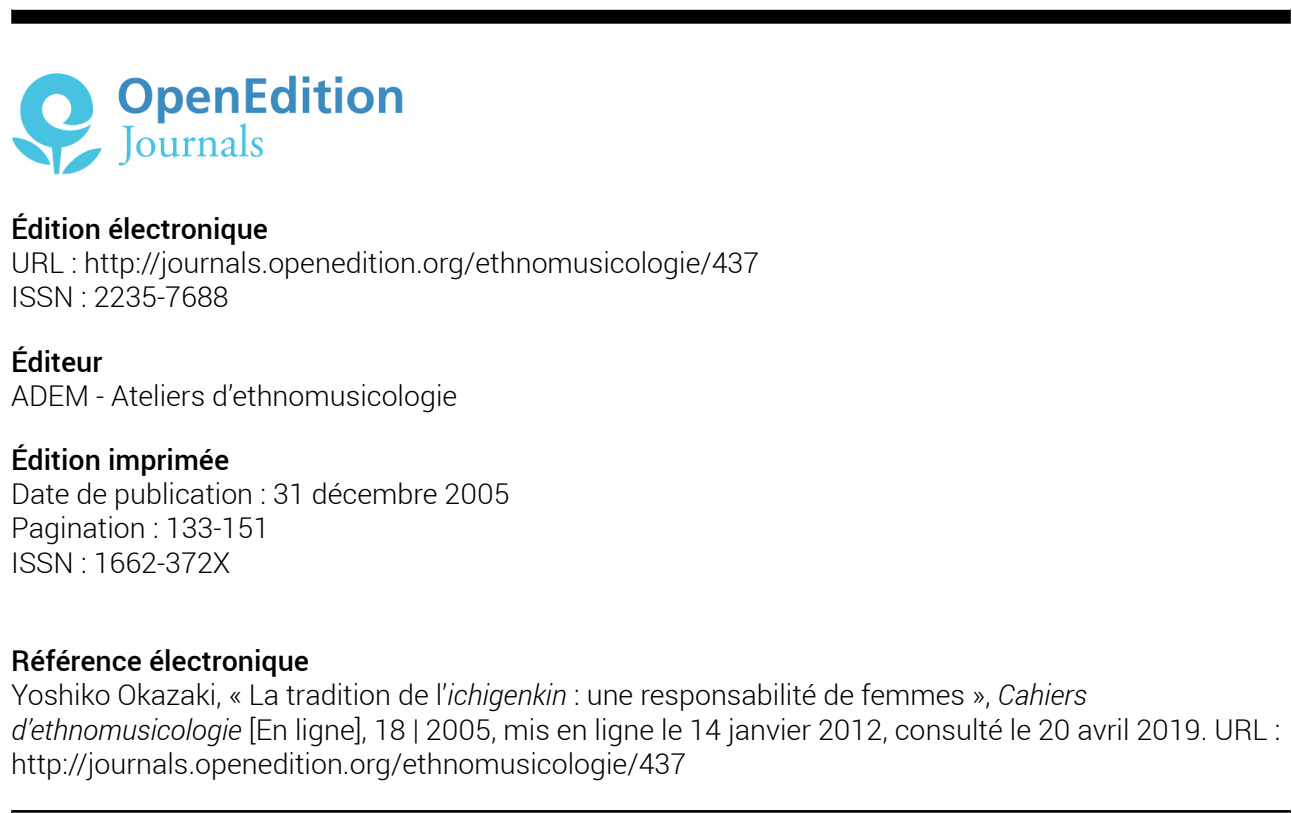

Ce document a été généré automatiquement le 20 avril 2019.

Tous droits réservés 


\title{
La tradition de l'ichigenkin : une responsabilité de femmes
}

\author{
Yoshiko Okazaki
}

Traduction : Isabelle Schulte-Tenckhoff

1 Le présent article aborde la tradition musicale de la cithare monocorde japonaise appelée ichigenkin. Jadis l'apanage des guerriers, des moines et des hommes de lettres, le jeu de cet instrument est, à présent, assuré et développé principalement par les femmes. Pratiqué par quelque cinq cents interprètes au plus, l'ichigenkin est un instrumentrare, relégué à la marge des principaux courants de la musique traditionnelle japonaise, surtout si on le compare au koto, la cithare à treize cordes largement répandue et considérée comme l'instrument représentatif du Japon.

2 Certains genres de la musique traditionnelle japonaise - tels que koto, shamisen, biwa et shakuhachi - ont toujours été pratiqués par des groupes incluant des femmes. Dans d'autres - comme hauta, kouta et kokyoku - la présence des femmes était même prédominante. La particularité de l'ichigenkin est qu'il atteste le glissement d'une tradition purement masculine à une tradition presque exclusivement féminine entre la fin du XIXe et le début du XX $X^{e}$ siècle ${ }^{1}$. La charte généalogique (fig. 1) indique les protagonistes du jeu de l'ichigenkin ayant transmis la tradition au long de son histoire. On dit que, dans sa forme actuelle, cette tradition remonte au moine zen Kakuho (1778-1815). 
Fig. 1 : Charte généalogique des joueurs et joueuses d'ichigenkin.

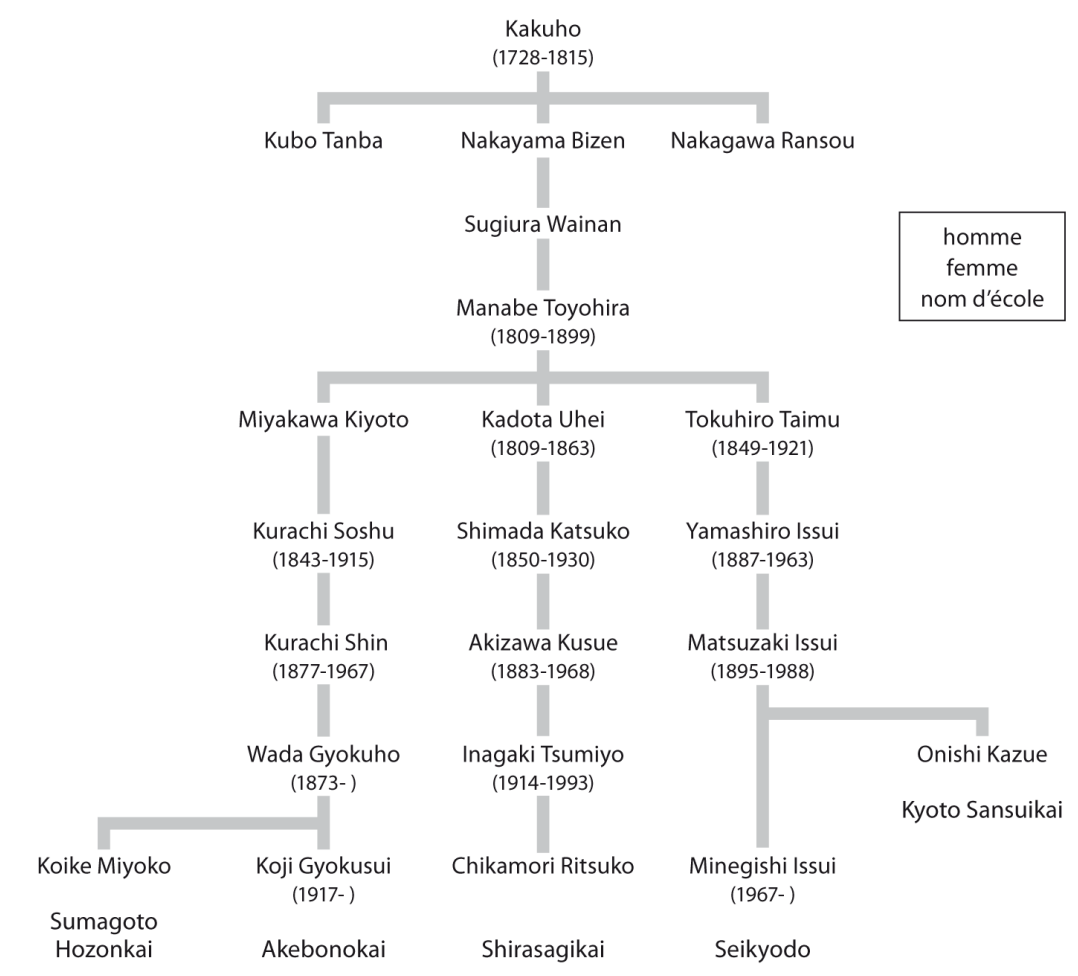

Cette charte est basée sur Onishi Kazue (1975 : 32). Les noms sont sélectifs, et les détails de la généalogie diffèrent selon les écoles, bien que toutes reconnaissent le rôle fondateur de Kakuho.

3 Comme l'indique notre charte, jusqu'à la quatrième génération depuis Kakuho, tous les noms sont masculins (indiqués par des caractères italiques); dès la cinquième, à l'époque de la restauration Meiji (1868), les noms se féminisent graduellement. Depuis lors et jusqu'à nos jours, ce sont presque exclusivement les femmes qui se consacrent au jeu de l' ichigenkin. Or, bien qu'on ne trouve guère d'interprète masculin, certaines écoles d' ichigenkin (appelées ryuuha) sont dirigées par des hommes ${ }^{2}$ - cette fonction étant désignée par le terme iemoto (littéralement : chef de la maison).

4 J'en profite pour mieux expliquer les termes iemoto et ryuuha car, au Japon, de nombreuses formes d'art, y compris la musique traditionnelle, la danse, le théâtre kabuki, les cérémonies du thé, l'art de l'arrangement floral et, plus récemment, le chant populaire, sont transmises à travers un système appelé ryuuha ou iemoto (ces deux termes étant ici interchangeables). En vertu de ce système, instauré sous la dynastie Edo (1603-1868), un maître et ses disciples forment une école structurée hiérarchiquement, où sont enseignés les traditions, les styles et la philosophie présidant à la performance. Les disciples apprennent un répertoire standard, copiant fidèlement la technique et le style de jeu du iemoto, la tradition se transmettant ainsi de génération en génération au sein de l'école ${ }^{3}$.

5 L'apparition du système du iemoto coïncide avec le moment où les femmes commencent à jouer un rôle dans les diverses traditions de l'ichigenkin. La concomitance dans le temps entre une féminisation radicale de l'interprétation et une normalisation des styles de jeu soulève plusieurs questions. Tout d'abord, on peut se demander de quelle manière et pour quelle raison les femmes se substituent aux hommes dans l'art de l'ichigenkin à ce moment 
particulier de l'histoire, tout en cherchant à savoir ce que l'on peut tirer de ce changement au sujet de la musique de l'ichigenkin et en quoi le jeu féminin diffère du masculin. Ensuite, il s'agit de savoir comment les changements relatifs à l'histoire et au genre se rapportent à la musique de ichigenkin et comment les femmes pratiquant aujourd'hui l'ichigenkin perçoivent la musique jadis interprétée par les hommes. Enfin, sur la base d'une interrogation soulevée dans l'ouvrage Women and Music in Cross-Cultural Perspective (Robertson 1987 : 245),on peut se demander si le jeu musical des femmes et celui des hommes visent des finalités différentes ou s'il ne sont pas plutôt motivés par les mêmes besoins d'ordre culturel.

6 Le présent article ne peut offrir qu'un traitement préliminaire de ces questions. Il existe actuellement au moins huit ou neuf écoles de ichigenkin. Dans ce qui va suivre, on s'intéressera à l'une d'entre elles, connue sous le nom de Seikyodo. Ma recherche se base sur des entretiens avec Minegishi, Issui, directrice de l'école, avec sa kõken ("gardienne »), Saito, Ichiyo, et avec les élèves de l'école. Mais auparavant, j'aimerais décrire brièvement l'histoire et la facture de l'instrument en question.

\section{L'ichigenkin}

Une seule corde en soie est tendue sur une table d'harmonie en bois de paulownia d'une épaisseur de $2 \mathrm{~cm}$ environ. Longue d'un mètre et large de $10 \mathrm{~cm}$, la table d'harmonie est munie d'un petit chevalet auquel est fixée une cheville. C'est un instrument simple, qui ressemble un peu au shakuhachi, lequel est fait d'un tube de bambou dont l'embouchure porte une encoche découpée selon un angle particulier. Or, si l'un et l'autre de ces instruments sont de facture simple, leur musique ne l'est de loin pas, car ils permettent tous deux d'émettre des sonorités fort subtiles et délicates par le recours à des techniques de jeu particulières.

8 Pour jouer de l'ichigenkin, il faut porter deux épaisses bagues d'ivoire creux (ou découpées dans un tube de bambou) appelées rokan, la plus courte sur l'index de la main droite, la plus longue sur le majeur de la main gauche. Avec la rokan de gauche, l'interprète touche légèrement la position appropriée sur la corde, tandis qu'elle la pince avec la rokan de droite. Les douze positions des doigts, appelées ki, sont indiquées sur la table d'harmonie. Dans le passé, l'ichigenkin était placé sur les genoux du musicien assis par terre, à l'instar des joueurs coréens du kayagum. De nos jours, on se sert d'un pupitre en bois, dont le matériau et la facture sont soigneusement sélectionnés pour assurer la meilleure qualité acoustique.

9 Quant à l'origine de l'ichigenkin, on ne dispose d'aucune source historique à proprement parler, hormis quelques légendes évoquant l'apparition de l'instrument et les personnages impliqués. Voici les récits les plus fréquemment racontés :

- En juillet 799, un Indien en habit de moine erre en portant sur l'épaule un instrument monocorde sur lequel il joue constamment une mélodie triste ${ }^{4}$.

- Au début de l'ère Héian, au IV e siècle, le poète Ariwara no Yukihira, qui est aussi le frère d'un sujet de l'empereur exilé à Suma (près d'Osaka), retire un bout de planche de son toit, y attache une ligne de pêche (ou un bout de ficelle de son chapeau, d'après une autre version), en confectionne une cithare monocorde et en joue pour consoler son chagrin et son trouble.

- Il existe une cithare monocorde en Chine, donnée en cadeau au roi de Ryukyu (l'actuel Okinawa). 
- Ces légendes offrent une série d'indices quant à la manière dont les joueurs de l'ichigenkin conçoivent les éléments essentiels de leur tradition, à savoir :

- le joueur de ichigenkin est un personnage solitaire qui se tient à l'écart du monde profane $(1,2)$;

- on joue de l'instrument pour se réconforter, non pour divertir un public $(1,2)$;

- l'ichigenkin provient de l'extérieur du Japon $(1,3)$;

- les joueurs de ichigenkin appartiennent à la noblesse et au clergé $(1,2)$;

- aucune de ces légendes - peu nombreuses, il est vrai - ne mentionne une femme jouant de l'ichigenkin $(1,2,3)$;

- le jeu de l'ichigenkin sert toujours à accompagner le chant, si bien qu'il appartient à la catégorie de musique vocale dont les paroles évoquent principalement la nature et la vie humaine ; ce n'est que récemment qu'on a vu l'apparition de compositions nouvelles à caractère purement instrumental.

\section{Les premiers maîtres de l'ichigenkin}

\section{Kakuho (1728-1815)} haut au sujet du fondateur de la tradition de l'ichigenkin évoquent un moine zen du nom de Kakuho (1729-1815). Rattaché au temple Kongorin à Kawachi (à l'est de l'actuel Osaka), Kakuho vit une existence dépouillée dans une retraite à la montagne. Il enseigne l'ichigenkin à ses visiteurs, la plupart d'entre eux appartenant à la féodalité guerrière et au groupe des hommes lettrés. Ceux-ci finissent par répandre la tradition de l'ichigenkin en d'autres régions du Japon. On dit que Kakuho est l'auteur des compositions les plus célèbres pour ichigenkin, soit «Imayo » et « Suma ». Non seulement Kakuho enseigne les techniques de jeu de l'ichigenkin, mais il cherche aussi à inculquer des valeurs morales aux musiciens (Seiha Seikyoku Shirasagikai $2000: 69$ ).

Parmi les enseignements légués par Kakuho, on retient les suivants :

- Vide-toi quand tu joues de l'ichigenkin.

- Chante et joue de l'ichigenkin en ayant l'esprit sain.

- Tiens-toi droit devant l'ichigenkin lorsque tu pinces la corde pour te maintenir en bonne santé.

- Joue régulièrement : il n'y a rien de mieux que la musique de l'ichigenkin pour se tenir à l'écart du monde.

- Ne joue jamais à la manière d'un enfant : bien que ténu et fragile, le son de l'ichigenkin chasse les ennuis et les soucis de ce monde et libère l'esprit ; instrument véritable,

l'ichigenkin n'est pas pour la frime et les lubies ; joues-en simplement, régulièrement et à cœur joie.

Les enseignements de Kakuho, visant à rehausser la spiritualité par l'étude de l'ichigenkin semblent évoquer le confucianisme ou les enseignements religieux du bouddhisme. En répandant la tradition de l'ichigenkin à travers le Japon, les disciples de Kakuho des deuxième et troisième générations - tous des hommes et appartenant, pour la plupart, à la noblesse guerrière ou lettrée - ont en même temps transmis l'esprit et les enseignements du maître. 


\section{Manabe Toyohira (1809-1999)}

13 Parmi les disciples de Kakuho, on trouve plusieurs maîtres réputés d'écoles de l'ichigenkin, dont le plus célèbre et influent est Manabe Toyohira, qui appartient à la troisième génération et forme de nombreux disciples venant des différentes régions $\mathrm{du}$ Japon. Son éminence particulière tient au fait qu'il a enseigné l'instrument à la plupart des fondateurs actuels d'écoles d'ichigenkin. Manabe Toyahira naît dans une famille de prêtres shintoïstes. En plus d'être un maître de l'ichigenkin, il est poète, érudit et artiste. Il renouvelle l'instrument et compose quantité de nouvelles pièces. En effet, près de la moitié du répertoire actuel de l'ichigenkin est attribuée à Manabe.

\section{Tokuhiro Taimu (1849-1921)}

14 Un disciple de Manabe est Tokuhiro Taimu, fondateur de l'école Seikyodo Ichigenkin ${ }^{5}$. Il connaît la fin de la période Edo (1603-1868), celle des Meiji (1868-1912) et le début de l'ère Showa (1912). Avec le déclin de la musique de l'ichigenkin sous l'effet de profonds bouleversements au sein de la société japonaise, Tokuhiro Taimu cherche à revitaliser la tradition en améliorant la facture de l'instrument, en élaborant un nouveau système de notation et en créant de nouvelles compositions. L'une de ses œuvres maîtresses est Hakusensou ("Méditation à la montagne »), expression d'une croyance selon laquelle l'harmonie entre les êtres humains et la nature mène à l'immortalitét. Adepte du bouddhisme zen, il se retire périodiquement dans des caves zen pour la méditation, toujours accompagné de son ichigenkin - le jeu de cet instrument faisant partie intégrante des exercices spirituels. Il dit notamment: «Evite de jouer de l'ichigenkin simplement pour faire étalage de ta technique. Ne recherche jamais la célébrité ».

D'après Tokuhiro Taimu, la valeur du jeu de l'ichigenkin réside dans les caractéristiques suivantes (Sansuikai $1985: 4$ ) :

- le jeu de l'ichigenkin fait ressortir la bonté naturelle de l'homme et affine les traits de visage ;

- l'ichigenkin est à l'origine de sonorités et de mélodies d'une grande beauté ;

- l'ichigenkin émet des notes transparentes et pures;

- l'ichigenkin produit des expressions hautement délicates ;

- l'ichigenkin est de facture élégante ;

- l'ichigenkin est facile à transporter ;

- l'ichigenkin n'est pas onéreux ;

- l'ichigenkin est facile à apprendre ;

- le jeu de l'ichigenkin renforce la fibre morale;

- l'ichigenkin convient à la musique destinée aux dieux et au Bouddha.

Tokuhiro n'a pas de fils, mais trois filles, qu'il n'hésite pas à désigner comme ses successeurs. Sa deuxième fille devient la deuxième iemoto, l'aînée étant décédée à un jeune âge ; puis sa troisième fille devient la troisième. Tokuhiro Taimu semble avoir une grande largesse d'esprit, bien que son style de vie soit plutôt austère et solitaire. Non seulement il lègue la tradition à une femme, mais il encourage encore la transmission du jeu de l'ichigenkin aux filles et aux femmes dans le cadre de leur éducation générale. Pour finir, il l'introduit au programme de l'enseignement obligatoire (Ohnishi 1975 : 33). C'est sous son égide que le sexe des interprètes d'ichigenkin va changer, de même que la finalité 
du jeu de cet instrument. En 1954, Tokuhiro Taimu est nommé « Trésor culturel national intangible $»^{7}$.

Les quatrième et cinquième générations après Kakuho - c'est-à-dire vers 1900 (voir fig. 1) - représentent donc un vrai tournant dans la tradition musicale de l'ichigenkin. C'est l'époque où la pratique de l'ichigenkin atteste un glissement d'une tradition exclusivement masculine à une tradition féminine, qui affecte à son tour la nature de la musique. Jadis élément de l'entraînement spirituel des guerriers, l'ichigenkin s'est intégré à la culture et à l'éducation des femmes. Cette métamorphose va de pair avec la transformation plus générale de la société japonaise, qui se manifeste dès la fin de l'ère Edo (1603-1868) et jusqu'au début de l'ère Meiji (1868-1921).

\section{Un tournant historique}

$18 \mathrm{Au}$ Japon, la fin du XIX ${ }^{\mathrm{e}}$ siècle fut une époque de profonds remous d'ordres politique, économique, social et culturel. Ainsi, la noblesse guerrière, qui s'était maintenue au pouvoir pendant près de sept cents ans depuis la fin du XII ${ }^{e}$ siècle, se voit privée de son rôle historique. Grâce à une période prolongée de paix régnant aux XVIII ${ }^{e}$ et XIX ${ }^{e}$ siècles, les marchands sont en mesure d'asseoir un pouvoir économique qui dépasse de loin celui de la classe guerrière, dont la prééminence s'en trouve définitivement ébranlée.

En 1868, le gouvernement Meiji nouvellement créé abolit la féodalité et les anciennes distinctions claniques (noblesse guerrière, paysannerie, classes artisanale et marchande, par ordre de rang décroissant), et tous les citoyens sont déclarés égaux, du moins aux termes de la loi. En outre, une série de réformes sociales est adoptée : création d'un système scolaire moderne, suppression des privilèges socio-économiques de la noblesse guerrière, en plus d'un effort généralisé de s'accommoder à la culture occidentale. Le confucianisme, pratiqué au Japon depuis le début du $\mathrm{VI}^{\mathrm{e}}$ siècle et parvenu à son apogée pendant l'ère Edo, a façonné tout le système des valeurs et la vision du monde des Japonais. Mais cette culture millénaire est désormais concurrencée par l'idéologie occidentale de la liberté, de l'individualisme, de l'égalité et de l'autonomie.

Comme on peut s'y attendre, la tradition musicale de l'ichigenkin n'échappe pas à l'emprise de ce défi. Tout d'abord, le type de musique pratiqué, par exemple, par les guerriers ne pouvait pas sortir indemne de cette transformation des valeurs et de l'organisation sociale, étant donné l'abolition de la noblesse guerrière tout court. Par ailleurs, une tradition vouée exclusivement à l'entraînement spirituel, imbue de l'esprit guerrier et marquée par la pensée confucianiste et zen, ne peut guère se maintenir alors que le reste de la société japonaise avance à grands pas vers l'occidentalisation et la modernisation ${ }^{8}$.

21 Comme le jeu de l'ichigenkin était à l'origine l'apanage exclusif des guerriers et des lettrés, l'abolition de la féodalité conduit tout naturellement à un déclin de cette tradition musicale. Or, les bouleversements sociaux qui ont marqué la fin du XIXe siècle ont, en même temps, levé les restrictions pesant sur les femmes dans le domaine des arts du spectacle et contribué à élargir l'éducation féminine en général. A l'époque, les filles et les femmes de bonne famille étaient formées à l'art de l'arrangement floral, à la cérémonie des arômes, à la cérémonie du thé, ainsi qu'à la musique et à la danse. Quant à la musique, le jeu de la cithare à treize cordes koto est, à l'époque, exclusivement réservé aux aveugles de la région de Kochi (une préfecture située au sud-ouest du Japon), si bien que nombre 
de femmes voyantes cherchent à pratiquer d'autres instruments à cordes comme l' ichigenkin (Seiha SeikyokuShirasagikai 2000: 75). Il est probable que la discipline rigoureuse et les valeurs associées au jeu de l'ichigenkin attirent les femmes de l'époque, préoccupées par la sauvegarde de la culture japonaise traditionnelle.

\section{Les joueuses d'ichigenkin}

\section{Shimada Katsuko (1850-1930)}

Le premier nom de femme apparaissant dans la tradition jusque-là purement masculine de l'ichigenkin est celui de Shimada Katsuko, originaire d'une famille appartenant à la noblesse guerrière. A l'âge de huit ans, elle commence à prendre des leçons d'ichigenkin auprès de Kadota Uhei, un disciple réputé de Manabe Toyohira (troisième génération après Kakuho). Elle est l'une de ces femmes voyantes de la région de Kochi qui choisissent l'apprentissage de l'ichigenkin. A la mort de Kadota, elle devient l'élève de Manabe Toyohira, le célèbre maître de Kadota. Bien qu'elle interrompe la pratique de l'ichigenkin après son mariage, elle la reprend une décennie plus tard et crée l'école Shimada, fréquentée par de nombreux élèves, principalement des femmes et des filles. En 1899, elle crée l'école d'eichigenkin du nom de Kochi Seifukai, où elle aura en tout quelque 370 élèves, principalement de sexe féminin, jusqu'à sa mort à l'âge de 81 ans.

Shimada est intransigeante quant au comportement de ses élèves; elle leur apprend comment ouvrir et fermer les portes coulissantes, comment enlever leurs chaussures à l'entrée de la maison, comment gérer le temps au cours de la leçon. L'observation de telles règles fait partie intégrante de l'apprentissage réussi de l'ichigenkin, au même titre que la discipline est essentielle au jeu de l'ichigenkin. Les enseignements de Shimada ont été transmis à l'école Shirasagikai, qui s'est développée dans la région de Kochi, et dont la tradition est réputée simple et sans prétention. Quelque vingt membres ont assisté aux célébrations du $55^{\mathrm{e}}$ anniversaire de l'école, en 2000.

\section{Yamashiro et Matsuzaki}

D'autres noms féminins de notre tableau renvoient aux deux filles de Tokuhiro, Yamashiro et Matsuzaki, respectivement les deuxième et troisième successeurs de l'école de Seikyodo Ichigenkin. Matsuzaki est par ailleurs l'arrière-grand-mère de l'actuelle iemoto, Minegishi Issui. Comme je l'ai mentionné plus haut, c'est là le tournant historique marquant la reprise par les femmes d'une tradition jadis purement masculine. Pourquoi et comment ce tournant s'est-il produit? De prime abord, on pourrait l'expliquer par le fait que Tokuhiro, ayant seulement des filles, était de ce fait privé d'un héritier mâle à qui transmettre la tradition. Mais s'il était convaincu que le iemoto devait être un homme, il aurait pu choisir un successeur parmi ses disciples masculins, en dehors du groupe de ses consanguins. C'est donc pour des raisons plutôt positives qu'il semble avoir choisi d'enseigner l'ichigenkin aux fillettes et aux femmes, à savoir pour parfaire leur éducation à la fois générale et morale. Ses filles, ayant appris l'esprit et la technique de Seikyodo Ichigenkin de leur père à la maison, vont transmettent ceux-ci à leurs élèves. Yamashiro fut nommée " Trésor culturel national intangible » en 1954, et Matsuzaki en 1977. Ainsi, les deux iemoto femmes de la Seikyodo Ichigenkin sont officiellement reconnues comme des représentantes importantes du patrimoine culturel japonais. 
Fig. 2 : Minegishi Issui jouant de l'ichigenkin

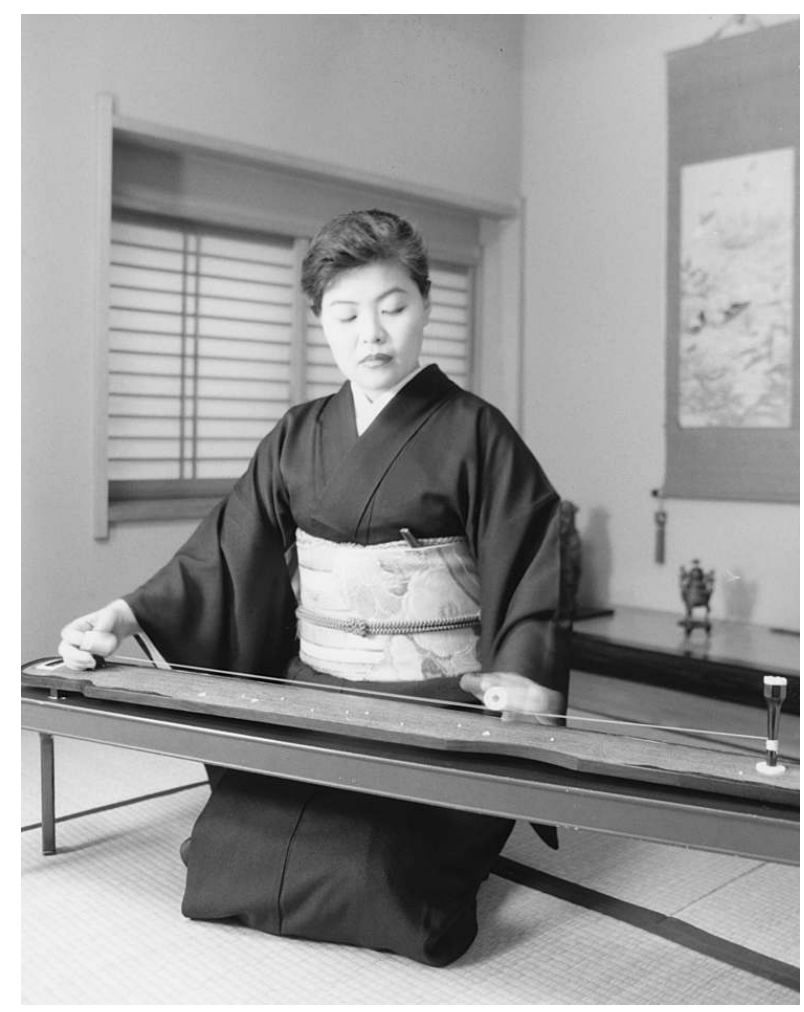

(photo : Minegishi Issui, juin 2001)

Il convient de mentionner une autre femme appartenant à la même génération, à savoir Kurachi Soshu (1843-1915), qui fonda l'école Moshiokai, plus tard rebaptisée Akebonokai.

\section{Les joueuses d'ichigenkin dans le Japon contemporain}

Lorsque le jeu de l'ichigenkin était l'apanage des hommes - guerriers, moines et lettrés de la société traditionnelle -, il était intégré à une éducation spirituelle austère et n'était pas destiné au divertissement d'un public. Une fois que les femmes prirent le relais, elles en redéfinirent l'objectif en l'intégrant à l'éducation générale des femmes et en le transformant en passe-temps récréatif. Dans ce qui va suivre, une place centrale revient à Minegishi Issui, la directrice de l'école Seikyodo Ichigenkin. Deux questions guideront ma présentation : la nature de la musique d'ichigenkin se trouve-t-elle altérée par ses objectifs redéfinis? et comment les interprètes féminines conçoivent-elles aujourd'hui l'actualité et l'avenir du ichigenkin?

\section{Minegishi Issui, directrice (iemoto) de l'école Seikyodo Ichigenkin}

27 Je m'intéresserai ici à la manière dont Minegishi Issui a enrichi la tradition en y ajoutant de nouveaux éléments, tout en préservant les éléments plus anciens requis par le système iemoto. En 1989, Issui est nommée quatrième successeur de l'école Seikyodo Ichigenkin. C'est une femme exceptionnelle dans le sens où on lui confère cette responsabilité alors qu'elle n'a que 21 ans, par décret de son arrière-grand-mère, Matsuzaki Issui, la troisième fille et troisième successeur de Tokuhiro Taimu. C'est un choix inattendu car il aurait tout 
aussi bien pu porter sur l'une ou l'autre des disciples de Matsuzaki ayant davantage d'expérience parce que plus âgée. Etant donné la jeunesse d'Issui, Matsuzaki nomme Saito Ichiyo - l'une de ses disciples ayant maîtrisé le jeu de l'ichigenkin et qui, souvent, la remplace dans ses cours - pour seconder la nouvelle iemoto dans sa tâche de perpétuer correctement la tradition. Ainsi, Saito Ichiyo est appelée à assurer que la jeune iemoto maîtrise complètement la tradition de l'école Seikyodo Ichigenkin dans l'esprit du fondateur, en préservant notamment sa philosophie quant au jeu de l'ichigenkin.

La nomination de la jeune iemoto suscite de nombreuses demandes de concerts d' ichigenkin, non seulement pour des rites aux sanctuaires et aux temples et pour des démonstrations scolaires, mais aussi pour des tournées au Japon et à l'étranger, y compris aux Etats-Unis, en Allemagne, en ex-Tchécoslovaquie, en France, en Italie, en Turquie et au Canada.

Tout en honorant ces nombreuses demandes, Issui rencontre quelques difficultés au cours des premières années suivant son entrée en fonction, à un moment où elle ne maitrise pas encore toute la signification de la musique de l'ichigenkin. Elle est fortement sollicitée à représenter l'école et à assumer son rôle de directrice face à des personnes dont certaines sont nettement plus âgées qu'elle. En un sens, elle partage le sentiment de nombreux jeunes qui ont de la peine à s'intéresser à une musique quelque peu monotone et assortie de vers anciens. Mais, grâce à des études plus poussées et à la pratique de l'instrument, avec aussi l'aide de divers collègues musiciens, elle finit par être convaincue que sa tâche ne consiste pas simplement à maintenir la tradition (principale responsabilité du iemoto), mais qu'elle doit aussi chercher à en transcender les limites et à élaborer une nouvelle tradition. Dans ce processus, un rôle particulier revient aux collègues qu'elle rencontre dans le cadre du NHK Hogaku Ginosha Ikuseikai, un programme de formation mené sous l'égide de la radio nationale, destiné aux jeunes interprètes d'instruments de musique traditionnels. En fait, le NHK Hogaku Ginosha Ikuseikai offre une éducation destinée à resserrer les liens entre différents genres et écoles au sein des musiques traditionnelles du Japon, qui n'auraient autrement pas eu la possibilité d'interagir. L'accent est mis sur l'apprentissage en des ensembles faisant intervenir différents instruments traditionnels. Issui est fortement inspirée par de nombreux spécialistes qui, comme elle, cherchent à incorporer de nouvelles formes d'expressions à la musique traditionnelle du Japon.

Consciente du message d'intériorité du fondateur - «Ne fais jamais étalage de ta technique»- Minegishi affirme vouloir suivre deux approches: l'une consiste à maintenir le caractère dépouillé de la musique de l'ichigenkin, et l'autre à explorer davantage le potentiel expressif du monocorde ${ }^{9}$. D'après elle, il faut absolument rendre l' ichigenkin plus attractif pour un public contemporain, en particulier auprès de la jeunesse. Il convient donc d'inclure de nouvelles compositions au répertoire et de promouvoir de nouveaux styles dans la musique de l'ichigenkin. La difficulté vient du fait que la créativité ne doit pas porter pas atteinte à l'austérité et à la spiritualité inhérentes à cette musique.

31 Ayant elle-même peu d'idées concrètes sur la manière de réaliser ces objectifs, elle se laisse inspirer par quelques connaisseurs, hommes et femmes. Tout d'abord par sa koken ("gardienne ») Saito Ichiyo, laquelle est très stricte dans la transmission de la tradition de l'école, mais en même temps assez ouverte aux idées nouvelles que Issui se propose de mettre en pratique. Ichiyo a elle-même composé de nouvelles pièces pour ichigenkin et arrangé de la musique provenant d'autres genres instrumentaux. Mais ces morceaux entrent dans le cadre d'expérimentations et ne sont pas censés outrepasser les limites traditionnelles de l'école. Certaines autres initiatives lancées par Issui vont cependant au- 
delà de la tradition de la Seikyodo ; c'est le cas du jeu de l'ichigenkin conjointement avec des instruments complètement différents, du recours à des poèmes étrangers et du développement de l'improvisation en cours de performance.

En 1996 par exemple, Issui donne un concert original avec une collègue joueuse du biwa (luth piriforme). La combinaison du biwa et de l'ichigenkin est plutôt inhabituelle, ne faisant sens que parce que les deux instruments sont joués en accompagnement de chants à contenu plutôt sérieux. Elle avait choisi des poèmes provenant des traditions amérindienne et inuit : la « Formule magique pour rendre l'ennemi paisible » des Navajos, le chant à l'aurore des Apaches Mescalero, les «Paroles magiques et autres paroles magiques » des Inuit, le "Chant de l'esprit gardien » des Nez Percés - tous traduits en japonais. Cette tentative de jouer de ces instruments sur des vers provenant de tradition littéraires non japonaises, mais néanmoins dotées d'un caractère spirituel et expressif marqué. Le programme comportait aussi des pièces du répertoire traditionnel (honkyoku) de chacun des deux instruments.

Un concert organisé en 1996 au Théâtre national à l'occasion du cent cinquantième anniversaire du fondateur de l'école Seikyodo Ichigenkin donne à celle-ci l'occasion de mettre en scène l'esprit guidant ses activités ainsi que sa conception de l'avenir. Le nombre et l'enchaînement des pièces, de même que l'interprète choisie pour chacune d'entre elles, a démontré un équilibre savamment dosé entre l'ancien et le nouveau. Le programme comprenait des honkyoku aussi bien que des gaikyoku - c'est-à-dire des pièces n'appartenant pas à la tradition de l'ichigenkin, empruntées par exemple au répertoire du koto ou du shamisen. Il comprenait également une pièce pour ichigenkin commanditée au célèbre musicien de gagaku Shiba Sukeyasu. Intitulée «Momiji no Nishiki» («Belle couleur des érables »), cette pièce met en musique le texte classique japonais «Ookagami » («Le grand miroir»); elle intègre des motifs rythmiques et mélodiques inhabituels dans la tradition de l'ichigenkin, comme des triolets et d'importants sauts mélodiques ${ }^{10}$.

En 1998, l'école commanda une composition à Takahashi Yuji, pianiste et compositeur de renommée internationale, en vue d'un concert organisé en hommage à l'ancien iemoto. Comme c'était la première fois qu'il composait pour l'ichigenkin - alors qu'il avait joué du shamisen et du koto, pour lesquels il avait également composé - Takahashi demanda immédiatement à Issui de lui donner des leçons d'ichigenkin, ce qui fit de lui l'un des rares élèves masculins de l'ichigenkin contemporain.

La composition réalisée par Takahashi comprend trois parties, basées chacune sur un poème : le premier, intitulé " $A$ une brise particulière ", est l'œuvre d'un poète japonais contemporain, Tachihara Michizo, le deuxième, "Miroir de la fleur », est de Zeami, le célèbre auteur du théâtre lyrique nô ; et le troisième, "We Can but Follow to the Sun ", fut écrit par l'Américaine Emily Dickinson. Dans l'ensemble, Takahashi s'est plié aux idiomes et aux styles traditionnels de l'ichigenkin, à l'exception de quelques techniques créées par lui, basées sur une transformation des lignes mélodiques ascendantes typiques appelées kororin (terme mnémonique rappelant une telle mélodie) en lignes descendantes. La deuxième partie de la pièce comprend un peu d'improvisation, assortie de la directive de « jouer librement, en choisissant n'importe quelle partie de la mélodie sur laquelle porter le regard au moment de chanter le poème ».

Le fait de bénéficier ainsi d'une reconnaissance de la part de compositeurs renommés revêt une importance capitale pour la tradition de l'ichigenkin. Mais il faut encore que les élèves de l'école manifestent un intérêt pour ces nouvelles pièces destinées à l'ichigenkin, 
afin qu'une nouvelle tradition se crée. Un tel intérêt existe, en effet, pour une nouvelle composition de Shiba, créée il y a trois ans et déjà devenue un nouveau classique du répertoire. Mais Issui s'interroge sur la longévité de la composition de Takahashi, car elle est plutôt difficile à jouer pour les élèves ordinaires, spécialement la troisième partie en anglais ${ }^{11}$.

Une expérience plus radicale fut tentée lors d'un autre concert de 1998. A cette occasion, Issui s'est essayée à une improvisation sur l'ichigenkin, conjointement avec Randy RaineReisch. Ce musicien et compositeur canadien, joueur d'ichigenkin, possède quelque six cents instruments traditionnels du monde entier; il est un virtuose sur une dizaine d'entre eux, dont l'ichigenkin ${ }^{12}$. Sur la base d'une seule formule mélodique, le duo a joué de l'ichigenkin sous forme de questions et réponses basées sur une cellule mélodique de base.

La musique ou les instruments associés à un groupe particulier doté d'un certain statut social ou réservés à un certain contexte de jeu sont inévitablement marqués par le changement social. Dès lors, pour survivre, la musique ou l'instrument doit, pour ainsi dire, se chercher un contexte ou un milieu culturel différent. Sous l'effet de l'abolition de la noblesse guerrière, la tradition de l'ichigenkin a subi un glissement en termes de genre et de culture. Des femmes comme Shimada, Yamashro et Matsuzaki ont joué un rôle d'intermédiaires entre l'ancien univers des guerriers et le contexte actuel, même si certains aspects de leur esprit originel ont résisté au temps. A l'heure actuelle, une jeune directrice d'école comme Minegishi Issui travaille énergiquement à assurer à la fois la continuité et le renouvellement de la musique de l'ichigenkin.

Fig. 3 : Concert à la Seikyodo Ichigenkin, Tokyo

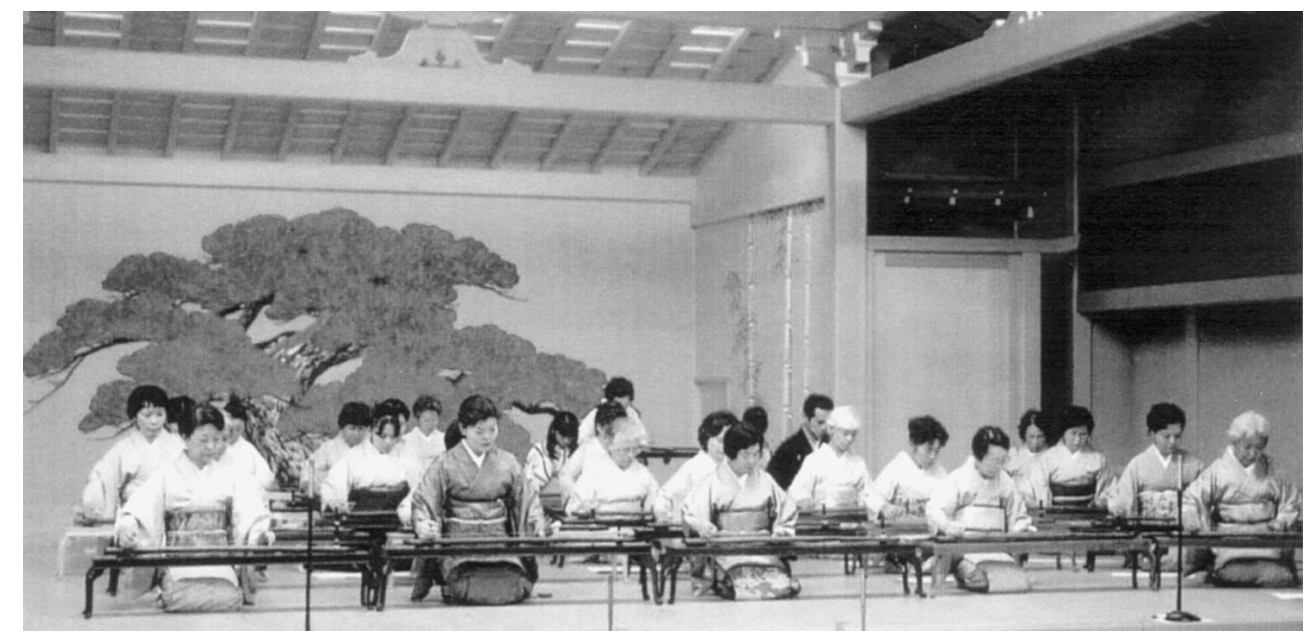

(photo : Minegishi Issui, 6 juin 2003).

\section{L' ichigenkin par les femmes}

Le chercheur Nishiyama Matsunosuke, spécialiste du système des iemoto, nous offre quelques éclaircissements quant à l'apport des femmes aux arts de la scène du $\mathrm{XX}^{\mathrm{e}}$ siècle. D'après lui, depuis le milieu du XXe siècle, au fur et à mesure que les femmes trouvent leur place dans le système en question, de nombreuses iemoto ont contribué à l'univers des arts japonais en relevant le défi que pose l'exigence de la préservation de l'esprit et de la versatilité des fondateurs originels, tout comme de ceux qui ont revitalisé la tradition. 
Plus généralement, ce phénomène a contribué à mettre en valeur les activités culturelles impliquant les femmes (Nishiyama 1989 : 192).

Minegishi Issui affirme à juste titre que l'arbitre de la tradition n'est pas le genre, mais la pérennité $^{13}$. Du point de vue théorique, la tradition de l'ichigenkin aurait pu être perpétuée par un groupe d'hommes dans le contexte contemporain, caractérisé par l'assouplissement de ses structures sociales. Mais, une fois disparue la finalité même de la performance de l'ichigenkin (c'est-à-dire l'entraînement spirituel du guerrier), un nouveau champ de signification devait émerger.

41 Le fait que l'esprit du guerrier ait été délaissé ne signifie pas pour autant que l'on s'écarte de la spiritualité traditionnelle japonaise. Un nouveau champ de signification a pu se construire, du moins partiellement, à partir de l'esprit fondamental associé à l'instrument. En effet, l'idéologie nationaliste qui émergea durant l'ère Meiji s'imposa dans le domaine non seulement politique, mais aussi culturel. D'un côté, on constate un mouvement vers une occidentalisation accélérée après plus de deux siècles d'isolationnisme. D'un autre côté, celle-ci se trouve contrebalancée par un mouvement de type nationaliste et impérialiste. L'éducation des femmes de bonne famille comprenait l'apprentissage de la culture traditionnelle du Japon, considérée comme aussi importante que des leçons de piano, pratique de plus en plus courante à l'époque. Bien que l'ichigenkin n'ait jamais été - et ne soit toujours pas - un art populaire largement pratiqué par les femmes, il a toujours existé un petit groupe de femmes attirées par cette musique et par l'instrument qui l'incarne. A l'instar de Minegishi Issui, ces femmes œuvrent de manière créative à sauvegarder et à développer la tradition. Ainsi pouvons-nous dire qu'à l'heure actuelle, la pérennité de l'esprit de l'ichigenkin est assurée par les femmes.

$\mathrm{Au}$ cours des entretiens que nous avons eus, Minegishi Saito et d'autres membres de l'école Seikyodo Ichigenkin ont partagé avec moi quelques réflexions sur la manière dont la musique pour ichigenkin était perçue et conceptualisée au sein de l'école ${ }^{14}$. Issui, tout comme Ichiyo, dit souvent que, lors des concerts à l'école, les interprètes amènent toujours un éventail, symbole de l'épée du guerrier, qu'elles placent à côté de l'instrument au moment de jouer. Elles racontent également qu'elles choisissent soigneusement le kimono revêtu en concert par les élèves pour qu'il représente les valeurs de la tradition: ses couleurs ne doivent pas être trop vives, et il ne faut pas non plus qu'il soit trop orné. Elles soulignent également que la tradition propre à l'école Seikyodo Ichigenkin ne doit jamais être présentée dans des lieux réservés au divertissement, notamment ceux où l'on consomme de l'alcool. Le sérieux et l'austérité : telles sont les valeurs essentielles de cette musique. C'est pourquoi Minegishi fait très attention aux paroles accompagnant les nouvelles compositions, ainsi qu'aux auteurs chargés de les créer.

Un autre thème récurrent est celui du caractère unique de l'ichigenkin en tant qu'instrument monocorde ${ }^{15}$; il n'en resterait rien si l'on supprimait cette seule corde. L'unicité de la corde elle-même est chargée de signification philosophique : elle renvoie aux possibilités d'une expression infiniment riche à partir de presque rien. Dans ce discours, qu'on rencontre également parmi les représentants d'autres expressions culturelles, on voit le désir de doter les traditions japonaises de significations uniques afin d'assurer leur survie dans une société de plus en plus occidentalisée.

Il est intéressant de relever que, parmi les musiciens professionnels ayant démontré un intérêt pour l'école Seikyodo Ichigenkin et ayant soutenu la tradition, on trouve des 
hommes comme Randy Raine-Reisch et Takahashi Yuuji. Alors que les restrictions traditionnelles liées au sexe de l'interprète n'ont plus la même importance aujourd'hui, que faut-il en conclure quant aux possibilités d'une carrière musicale? D'après Oikawa, l'un des élèves masculins de feu Matsuzaki, puis de Ichiyo, la raison pour laquelle le ichigenkin est surtout devenu populaire parmi les femmes doit être cherchée dans le fait que celles-ci disposent de davantage de temps libre ${ }^{16}$. Il est effectivement fort difficile de gagner sa vie en tant que joueur d'ichigenkin, à moins d'être un musicien confirmé.

Une des caractéristiques de l'ichigenkin est peut-être que ceux qui désirent apprendre à en jouer disent que c'est presque par hasard qu'ils ont été attirés par l'instrument et touchés par ses sonorités ; mais que, lorsqu'ils sont préoccupés ou tristes, le jeu les réconforte. Minegishi Issui raconte qu'elle n'oubliera jamais le moment où son arrière-grand-mère, Matsuzaki, troisième iemoto, se mit à jouer le matin du jour de funérailles de son mari défunt ${ }^{17}$. Sur le moment, Minegishi Issui était convaincue qu'une femme endeuillée avait besoin de jouer de cet instrument.

\section{Conclusion}

En guise de conclusion, j'aimerais soulever quelques questions susceptibles de guider la recherche à venir. Les études traitant de la musique et du genre, souvent axées sur les rapports de pouvoir entre hommes et femmes, sont-elles pertinentes pour analyser la tradition de l'ichigenkin? Dans quelle mesure et dans quel sens pouvons-nous rapporter cette tradition musicale à l'histoire du Japon et à la société japonaise actuelle ? L'étude du système des iemoto revêt ici une importance cruciale en ceci qu'il s'écarte parfois de la hiérarchie traditionnelle dont était empreint l'ordre social. Des femmes ont par exemple $\mathrm{pu}$ atteindre un statut social plus élevé grâce à leur apprentissage de l'ichigenkin; des maitres provenant des classes populaires ont enseigné à des disciples appartenant à la noblesse guerrière; et certaines femmes avaient le droit d'enseigner aux hommes (Nishiyama 1989 : 188). Lorsque la société japonaise s'est trouvée libérée - du moins en droit - des restrictions imposées par la féodalité et que les classes populaires se sont enrichies, les spécialistes des arts de la scène ont commencé à occuper une place importante dans la société (Nishiyama 1989 : 187-188). Nombre de iemoto, dont les élèves étaient presque tous des hommes, ont pu se produire devant un public composé de personnes de rang plus élevé, même s'ils n'appartenaient eux-mêmes qu'à la classe marchande ou à la paysannerie; dans certains cas, on les considérait, en tant que iemoto, comme étant de rang plus élevé que leurs disciples provenant de la noblesse guerrière (Nishiyama 1989: 188). Plutôt qu'une révolution par la force, on peut y voir une révolution de la vie culturelle ayant de quoi séduire les gens du commun auxquels la musique permettait d'échapper au système rigide des quatre classes (guerriers, paysans, artisans et marchands, par ordre décroissant) (Nishiyama 1989 : 184).

Un autre champ d'étude fécond a trait à la musique et à la psychologie féminine au sein de la société japonaise. Certaines interprètes d'ichigenkin abordent la musique en fonction de la capacité du ichigenkin à leur procurer du réconfort lorsqu'elles sont troublées ou tristes. A ce stade, il existe peu d'études sur le rapport entre performance musicale et émotions parmi les femmes japonaises. Que révèle la dimension affective du jeu de l' ichigenkin sur la vie des femmes ? De quelle manière la musique pour ichigenkin offre-t-elle une nouvelle liberté ou, inversement, impose-t-elle de nouvelles contraintes? Bien évidemment, il faut entreprendre des recherches plus approfondies que celle sur laquelle 
se fonde le présent texte. J'ajouterai que ce type de recherche exige que l'on analyse soigneusement des cas individuels plutôt que de chercher à exposer de grandes théories générales.

\section{BIBLIOGRAPHIE}

FUJIWARA Fuyutsugu c.840 Nihonkouki (Japanese History). n.p.

HUGHES David 1993 « Regional Studies: East Asia 2, Japan », in Helen Myers ed.: Ethnomusicology: Regional Studies and History. New York/London: Norton \& Co.: 345-362.

KIKKAWA Eishi et al. 1983 " Tokushu Josei to Hogaku » (Special Issue: « Women and Traditional Musics of Japan »), in Kikan Hogaku (Journal of Japanese Traditional Music) 36: 63-83.

KOSKOFF Ellen ed. 1987 Women and Music in Cross-Cultural Perspectives. Urbana and Champaign: University of Illinois Press.

MYERS Helen, ed. 1983 Ethnomusicology: Regional Studies and History. New York/London: Norton \& Co.

NISHIYAMA Matsunosuke 1989 Nihon no Bi to Dentou (Japanese Sense of Beauty and Tradition). Tokyo: Iwanami-shoten.

ONISHI Kazue 1975 Ichigenkin Sikyodo Shinpu Kaisetsu (An Introduction to the New Notations of the Seikydo Ichigenkin). Kyoto: n.p.

ROBERTSON Carol E. 1987 « Power and Gender in the Musical Experience », in Ellen Koskoff ed.: Women and Music in Cross-Cultural Perspectives. Urbana and Champaign: University of Illinois Press: 225-244.

SEIKYODO 1985 Sikyodo Ichigenkin Kaisetsu (An Introduction to Sikyodo Ichigenkin). Revised ed. Tokyo: Sansuikai.

Seikyoku Ichigenkin Shirasagi kai 2000 Hitosuji no Ito wa Nagarete (A Flow of the One String). Kochi: shirasagikai.

The Seikyodo Ichigenkin School 1994 The program notes of the Seikyodo Ichigenkin Concert at the Boston Conservatory. March 30.

\section{ANNEXES}

\section{Entretiens}

Ishizaka Issei, Tokyo, 5 juillet 2004.

Minegishi Issui, Tokyo, 25 juillet 2001, 29 septembre 2003, 8 avril 2004, 22 juin 2004.

Oikawa Takao, Saitama, 26 août 2004. 
Saito Ichiyo, Tokyo, 12 juillet 2002, 8 avril 2004.

Elèves de l'école Seikyodo Ichigenkin, Tokyo, 20 mai 2000.

\section{NOTES}

1. Pour plus d'informations, voir le vol. 36 (1983) de la revue japonaise Kikan Hogaku (Journal of Japanese Traditional Music). Il s'agit d'un numéro spécial consacré aux femmes dans la musique traditionnelle du Japon ; voir aussi notamment Kikkawa (1983).

2. L'une des écoles de ichigenkin dirigée par un homme, à savoir Takahashi Toru, est l'école Yu-i Ichigenkin (www.geocities.co.jp/MusicHall-Horn/9183/Yu-i-entlish.html; Takahashi Toru, 15 novembre 2004).

3. Le système iemoto a exercé une influence profonde sur la transmission des arts au Japon. A la tête d'une école, la ou le iemoto exerce souvent un pouvoir considérable, prenant toutes les décisions relatives aux styles de jeu, à l'accréditation des maîtres, et ainsi de suite (Hughes 1993 : 353).

4. Ce récit figure dans un recueil historique ancien, à savoir Nihonkooki, vol. 8 (Fujiwara, Fuyutsugu, vers 840).

5. Il est l'arrière-arrière-grand-père de Minegishi, Issui, l'actuel quatrième successeur (une femme).

6. D'après le programme du concert donné par l'école Seikyodo Ichigenkin au Conservatoire de Boston, le 30 mars 1994.

7. Il s'agit là d'une distinction attribuée par l'Agence japonaise des Affaires culturelles, selon un système instauré en 1950 afin d'assurer la sauvegarde du patrimoine culturel du Japon.

8. La secte zen a été l'épine dorsale de l'esprit guerrier. Sa méthode d'éducation spirituelle est basée sur la méditation religieuse et la quête d'une sérénité dont le jeu de l'ichigenkin est, précisément, la condition préalable.

9. Entretien le 29 septembre 2003, Tokyo.

10. Pour résoudre le problème technique posé par de tels écarts, ceux-ci sont joués par deux personnes qui se répartissent les notes basses et aiguës.

11. L'utilisation de l'anglais pour la troisième partie répond, selon Takahashi, au désir de montrer sa considération pour le public et les interprètes non japonais de l'ichigenkin.

12. Randy Raine-Reisch a été formé à l'ichigenkin d'abord par feu Yamada Isshi, directeur de la filiale Seikyodo de Hawaï, puis par Saito Ichiyo.

13. Entretien, le 8 avril 2004, Tokyo.

14. Entretiens, tous à Tokyo, avec Minegishi Issui le 29 juin 2004, avec Saito Ichiyo le 12 juillet 2002, avec Issei Ishizaka le 29 juin 2004, et avec des élèves de l'école Seikyodo Ichigenkin le 20 May 2001.

15. Entretiens, tous à Tokyo, avec Minegishi Issui le 29 juin 2004, avec Saito Ichiyo le 12 juillet 2002, avec Issei Ishizaka le 29 juin 2004, et avec des élèves de l'école Seikyodo Ichigenkin le 20 May 2001.

16. Entretien, le 26 août 2004, à Saitama.

17. Entretien du 25 juillet 2001. 


\section{RÉSUMÉS}

Cet article aborde la tradition musicale de la cithare monocorde japonaise appelée ichigenkin. Jadis apanage des guerriers, des moines et des lettrés, notamment entre la seconde moitié de l'ère Edo (1603-1868) et le début de l'ère Meiji (1868-1912), le jeu de cet instrument est, à présent, assuré et développé principalement par des femmes. L'article offre un traitement préliminaire des questions suivantes : de quelle manière et pour quelle raison les femmes se substituent aux hommes dans l'art de l'ichigenkin en ce moment particulier de l'histoire; et que ce signifie ce glissement pour la musique de l'ichigenkin? Après une brève description de l'histoire de l' ichigenkin, l'auteur nous permet de découvrir l'une de ses interprètes contemporaines. Elle explore en outre la manière dont se crée une tradition nouvelle bien que toujours fondée sur l'esprit originel de l'instrument, tout en évoquant le rôle d'interprètes masculins dans ce processus.

\section{AUTEURS}

\section{YOSHIKO OKAZAKI}

Yoshiko Okazaki est titulaire d'un doctorat en ethnomusicologie obtenu à l'Université de Californie, Los Angeles. Elle enseigne les cultures d'Asie et les musiques du monde à l'Université Sacred Heart, Tokyo, en tant que professeur au Département d'histoire et de sociologie, ainsi que l'ethnomusicologie à l'Université nationale des beaux-arts et de la musique, également à Tokyo. 